\title{
Enfants et parents
}

Les nouvelles de ce numéro ont été préparées par: Jean-Claude Dreyfus Serge Erlinger Axel Kahn Marc Peschanski

\begin{tabular}{|c|c|c|c|c|c|}
\hline \multicolumn{6}{|c|}{$\begin{array}{c}\text { Tableau I } \\
\text { SITUATION A L'ENQUETE }\end{array}$} \\
\hline & \multicolumn{5}{|c|}{ Age de l'enfant } \\
\hline & Ensemble & $0-4$ ans & 5.8 ans & 9.12 ans & 13.16 ans \\
\hline \multirow{8}{*}{$\begin{array}{l}\text { Père et mère } \\
\text { mariés } \\
\text { Père et mère } \\
\text { cohabitants } \\
\text { Mère + } \\
\text { un conjoint } \\
\text { Përe + } \\
\text { un conjoint } \\
\text { Mère seule } \\
\text { Père seul } \\
\text { Aucun parent } \\
\text { Inconnu }\end{array}$} & 828 & 849 & 857 & ב2? & 782 \\
\hline & 3.4 & & & 0.8 & 1.4 \\
\hline & 3.7 & 1,0 & 3.5 & 6,6 & 4.5 \\
\hline & 07 & 02 & 03 & 10 & 13 \\
\hline & 6.9 & 5,0 & 5.8 & 7.0 & 10.0 \\
\hline & 0.9 & 0.2 & 0,8 & & \\
\hline & 1.1 & 0.5 & 1.2 & 1.3 & 1.5 \\
\hline & & 0.4 & 0,2 & 0,3 & \\
\hline Total & 100.0 & 100,0 & 100,0 & 100,0 & 100.0 \\
\hline
\end{tabular}

\begin{tabular}{|c|c|c|c|c|c|}
\hline \multicolumn{6}{|c|}{$\begin{array}{c}\text { Tableau II } \\
\text { RELATIONS DES PARENTS } \\
\text { A LA NAISSANCE }\end{array}$} \\
\hline & \multicolumn{5}{|c|}{$\begin{array}{c}\text { Age de l'enfant } \\
\text { (et année de naissance) }\end{array}$} \\
\hline & Ensemble & \begin{tabular}{c|}
0.4 ans \\
$(1981$ \\
$-85)$
\end{tabular} & $\begin{array}{l}5.8 \text { ans } \\
\text { (1977 } \\
.80)\end{array}$ & $\begin{array}{c}9.12 \text { ans } \\
\text { (1973 } \\
.76)\end{array}$ & $\begin{array}{c}13-16 \text { ans } \\
(1969 \\
.72)\end{array}$ \\
\hline $\begin{array}{l}\text { Naissances } \\
\text { légitimes } \\
\text { d'après } \\
\text { l'état civil }\end{array}$ & 89.4 & 84.0 & 90,0 & 91,6 & 93,0 \\
\hline \multirow{3}{*}{$\begin{array}{l}\text { Parents } \\
\text { mariés } \\
\text { (ensemble) } \\
\text { Parents } \\
\text { cohabitants } \\
\text { Parents } \\
\text { vivant } \\
\text { séparément } \\
\text { Situation } \\
\text { inconnue }\end{array}$} & 89.5 & 85,0 & 90,4 & 89.8 & 93.5 \\
\hline & 2,5 & $\begin{array}{l}11,0 \\
3,3\end{array}$ & 2.0 & $\begin{array}{l}6.1 \\
2.9\end{array}$ & $\begin{array}{l}4.1 \\
1.7\end{array}$ \\
\hline & 0.8 & 0.7 & 0.9 & 1.2 & 0.7 \\
\hline Total & 100.0 & 100,0 & 100,0 & 100.0 & 100.0 \\
\hline
\end{tabular}

1. Bourguignon $\mathrm{O}$, Rallu JL, Théry I. Du divorce et des enfants (cahier $n^{\circ} 3$ ). Paris : INED-PUF, 1985.

2. Leridon H, Villeneuve-Gokalp C. Entre
Les changements de mœurs des dernières années retentissent sur la situation de nombreux enfants. Près d'un quart de naissances hors mariage, un divorce sur quatre mariages, l'augmentation du nombre des couples dont au moins un membre a été marié, font que beaucoup d'enfants sont séparés au moins d'un de leurs parents, et ont éventuellement à faire face à la formation d'un nouveau couple dans leur foyer. Pour la première fois en France une enquête a été réalisée en 1985 par l'INED et l'INSEE [1]. Elle a porté sur un échantillon représentatif (468l enfants) et des conclusions ont pu en être dégagees [2]. Les enfants étudiés ont de 0 à 16 ans; pour interpréter les résultats il faut distinguer les situations au moment de l'enquête et à la naissance.

A la date de l'enquête, une donnée essentielle ressort (Tableau I): $17 \%$ des enfants ne vivent pas avec leurs deux parents mariés, ce qui pour l'ensemble de la France représente environ 2200000 enfants. Mais parmi eux la situation est très différente selon qu'ils vivent avec leurs deux parents non mariés $(450000)$, une famille «recomposée », le plus souvent la mère avec un nouveau conjoint $(600000)$ ou, cas le plus fréquent, la famille uniparentale ( 1000000$)$, c'est-à-dire la mère seule dans $85 \%$ de ces cas.

Si l'on rapporte les données à la situation à la naissance les résultats apparaissent différents. En effet, si entre 1969 et 1985 plus de $10 \%$ des enfants sont nés hors mariage (et en proportion croissante), moins de $3 \%$ sont nés dans une famille uniparentale, et la proportion n'en augmente pas (Tableau II). La montée des «naissances naturelles » est donc uniquement due à celle de l'union libre. Ce phénomène influet-il sur le devenir des enfants? Il faut savoir que la loi traite de façon différente les couples mariés ou non. Pour la mère la situation est la même : il suffit qu'elle soit désignée dans l'acte de naissance pour que soit créé le lien juridique. Pour exister, le père d'un enfant naturel doit le reconnaître; en 1985 la proportion des enfants reconnus était de $57 \%$ à la naissance pour dépasser $70 \%$ à la fin de la première année; elle continuera à augmenter lentement au fil des ans, pour atteindre sans doute comme par le passé environ $80 \%$. Parmi les enfants nés hors mariage, un sur cinq ne sera jamais reconnu.

Le dernier problème est celui des relations avec le parent absent. Il se pose pour $14 \%$ des enfants, et pour $20 \%$ entre 13 et 16 ans. Si les parents sont divorcés, dans $5 \%$ seulement des cas la garde est conjointe; pour les autres, elle est confiée à la mère dans $85 \%$ des cas. Si les parents ne sont pas mariés, la garde légale revient actuellement automatiquement à la mère *. Un des faits les plus importants qui ressort de l'enquête est que plus de la moitié des enfants $(54 \%)$ de parents séparés perdent toute relation suivie avec le parent non gardien, le père presque toujours. L'élément principal qui régira la relation ultérieure avec le père est constitué par les liens qui l'unissaient à la mère. Il est inutile d'insister en outre sur l'importance des conditions de la séparation pour que l'enfant conserve deux parents. En conclusion il faut rappeler que $97 \%$ de l'ensemble des enfants de moins de 16 ans vivent toujours avec au moins un de leurs parents et $80 \%$ avec leurs deux parents. Ces statistiques un peu sèches ont le mérite de substituer des données solides à des conjectures floues, et de pouvoir servir de base aux efforts faits pour améliorer le sort des enfants de parents séparés.

* Cette situation pourrait se modifier grâce à des changements de législation proposés en 1987. 